SUBJECT AREAS:

NEUROSCIENCE

WAKEFULNESS

Received

24 February 2014

Accepted

29 May 2014

Published

16 June 2014

Correspondence and requests for materials should be addressed to G.B. Igangbai@ nankai.edu.cn); X.Z. (zhaoxin@nankai.edu. cn) or X.Z.F. (xzfeng@ nankai.edu.cn)

* These authors contributed equally to this work.

\section{Behavioural screening of zebrafish using neuroactive traditional Chinese medicine prescriptions and biological targets}

\author{
Ya-Nan Wang ${ }^{1 *}$, Yuan-Yuan Hou ${ }^{2 *}$, Ming-Zhu Sun ${ }^{3 *}$, Chun-Yang Zhang ${ }^{3}$, Gang Bai ${ }^{2}$, Xin Zhao ${ }^{3}$ \\ \& Xi-Zeng Feng'
}

\begin{abstract}
${ }^{1}$ State Key Laboratory of Medicinal Chemical Biology, College of Life Science, Nankai University, Tianjin 300071, China, ${ }^{2}$ State Key Laboratory of Medicinal Chemical Biology, Tianjin Key Laboratory of Molecular Drug Research, College of Pharmacy, Nankai University, Tianjin 300071, China, ${ }^{3}$ The Institute of Robotics and Automatic Information Systems, Nankai University, Tianjin
\end{abstract} 300071, China.

The mechanism of the therapeutic action of antidepressants remains uncertain in traditional Chinese medicine (TCM). In this study, we selected 7 classical TCM prescriptions and utilised an automatic video-tracking system to monitor the rest/wake behaviour of larval zebrafish at 4 days post-fertilisation (dpf) for 48 hours. We found that the curative effects of the prescriptions were dose-dependent. K-means clustering was performed according to the shared behavioural phenotypes of the zebrafish. The results revealed that the rest/wake behavioural profiles induced by the same class of prescriptions were similar. A correlation analysis was conducted between the TCM prescriptions and the known compounds. The results showed that the TCM prescriptions correlated well with some well-known compounds. Therefore, we predicted that they may share a similar mechanism of action. This paper describes the first study to combine TCM research with zebrafish rest/wake behaviour in vivo and presents a powerful approach for the discovery of the mechanism of action of TCM prescriptions.

epression is a type of mood disorder. It is related to the normal emotions of sadness and bereavement, but it does not remit when the external causes of these emotions dissipate ${ }^{1}$. Depression is characterised by decreased activity, a significant and lasting low mood, and a slowing of thinking and cognitive function. At the same time, it often leads to some psychophysiological changes, such as disturbances in sleep and appetite, which considerably interfere with an individual's work and family relations ${ }^{2,3}$. Patients with depression have a high risk of suicide, and they have no ability to experience pleasure in their work or with their friends. Furthermore, the incidence and recurrence rates of the disease are high, and the onset age varies widely ${ }^{4}$. The World Health Organization states that depression is the second largest global health burden ${ }^{5}$. Although this disease has gained wide attention from scholars, to date, the aetiology and pathogenesis of depression are still not clear, and the diagnosis is largely based on the description of the symptoms ${ }^{6}$. In general, studies have shown that depression is associated with the biological, psychological, and social factors ${ }^{7-9}$. Currently, antidepressant drugs are the main methods used to treat depressive disorders and can effectively relieve depressed mood, which is accompanied by anxiety and somatic symptoms. In addition, psychological therapy, electroconvulsive therapy, and other physical therapies are also administered ${ }^{10,11}$. The most widely used antidepressants include tricyclic antidepressants (TCAs), monoamine oxidase inhibitors (MAOIs), selective serotonin reuptake inhibitors (SSRIs), and serotonin and norepinephrine reuptake inhibitors (SNRIs). These drugs are able to improve the depressed state at different levels; however, most of them have side effects ${ }^{12-14}$. The most commonly observed side effects associated with most antidepressant monotherapy groups are headache, nausea or vomiting, agitation, sedation, and sexual dysfunction ${ }^{15,16}$. In addition, drowsiness, fatigue, and anxiety have also been reported ${ }^{17}$. A number of studies have reported that antidepressants have cardio-toxic properties. For example, tricyclic antidepressants have effects on cardiac action potentials, which are highly cardio-toxic in overdose and may induce cardiovascular disease ${ }^{18-20}$. Therefore, many studies have focused on natural medicine, including traditional Chinese medicine (TCM).

TCM considers that the material bases of emotional activities are the "Five Internal Organs" and the "Qi and Blood". The emotional activities of an individual are closely associated with the asthenia and function of the internal organs, the Qi and blood, and the "Yin and Yang". The various external stimuli acting on the respective 
Zang-Fu organs lead to changes in the Qi and blood and the Yin and Yang of the Zang-Fu organs, thereby yielding various emotions ${ }^{21}$. In general, the stimulus of negative emotions, such as regret, will not result in the onset of disease. However, when a strong external stimulus is beyond the range within which a human body can regulate it, resulting in impaired functions of the Zang-Fu organs and a disorder of the Qi and blood, an excessive regret emotion will be generated, which will be a pathogenic factor ${ }^{22-24}$. Therefore, when treating depression, TCM starts at the whole-body level, considering not only the spiritual problems that resulted from the nervous system disorder of a patient but also the changes in the Zang-Fu organs and the Qi and blood. During the development of the disease, the treatment also evolves. Most TCM specialists consider that depression is mainly a disorder of the Yin and Yang, which results from Qi depression, thereby disturbing phlegm and blood stasis. The liver, heart, spleen, and kidney are the main disease sites ${ }^{25}$. The liver plays a principal role during the emotional changes of an individual, especially during the regulation of the various changes generated from emotional factors. If the catharsis function of the liver is impaired, stagnancy of the liver Qi will occur. A small stimulus will result in inadequate depression relief, low spirits, and taciturnity; thus, other organs of the body will also exhibit many pathogenic reactions ${ }^{26,27}$. Therefore, soothing the liver Qi, soothing the liver, and activating the spleen have gradually become the main methods of treating depression in $\mathrm{TCM}^{28-31}$. Furthermore, according to the different characteristics of the symptoms, tonic and tranquilising therapies are also frequently used for the treatment of depression. Specifically, the combined application of these therapies with chemical pharmaceuticals has yielded good curative effects clinically ${ }^{32-34}$. Although the clinical effects are significant, few studies on the use of TCM treatment for depression have been conducted. In this study, 7 classical prescriptions commonly used in various therapies were selected, and the effects of these compounds on the behaviour of the zebrafish were examined. The 7 prescriptions were Chai-Hu-Shu-Gan-San (prescription 1), Gan-Mai-Da-ZaoTang (prescription 2), Gui-Pi-Tang (prescription 3), Wen-DanTang (prescription 4), Tao-Hong-Si-Wu-Tang (prescription 5), Qi-Ju-Di-Huang-Wan (prescription 6), and Liu-Jun-Zi-Tang (prescription 7). The 1st prescription is often used to soothe the liver and Qi. The 2nd and 4th prescriptions have a tranquilising effect. The 3rd prescription is able to soothe the liver and activate the spleen. The 5th, 6th, and 7th prescriptions have a tonic function. Overall, these compounds improve depressed mood from four different perspectives.

As a vertebrate model organism, zebrafish have long been widely utilised for biomedical research, particularly in neuroscience ${ }^{35,36}$, drug discovery, and developmental and genetic research ${ }^{37-39}$. They present many advantages for the study of human disease and have become an excellent choice for behavioural experiments ${ }^{40-42}$; these advantages include the low maintenance costs, a short reproductive cycle, and rapid and synchronised development. In addition, they have a large number of offspring, and a single breeding pair can produce hundreds of fertilised eggs at a time. The embryos are large and transparent. Therefore, larval zebrafish are well-suited for largescale studies. Recently, they have been used for rapid and highthroughput screens for neuroactive and neurotoxic drugs because of the conservation of the nervous system between zebrafish larvae and mammals ${ }^{43}$. Compared with other animal models, zebrafish can be grown more easily in the laboratory.

\section{Results}

Establishment of a zebrafish rest/wake behavioural assay. In this study, we utilised a high-throughput, quantifiable approach to record the larval locomotor activity (Fig. 1A). Considering the known concentration effects of the investigated prescriptions, we conducted preliminary experiments to determine the appropriate concentration range and chose the final concentrations of $10,50,100$,
200, 500, 800, and $1000 \mu \mathrm{g} / \mathrm{mL}$ (Fig. 1B). All 7 TCM prescriptions in our study were antidepressants, and they have been used in the treatment of some mental illnesses. As described above, depression often leads to disturbances in sleep, including changes in the sleep structure, difficulty falling asleep, sleep maintenance disorders, insomnia or hypersomnia, and low sleep efficiency. An in-depth study of sleep disorders is significant for the clinical treatment and prognostic judgment of depression. The influences of different antidepressants are diverse. Some sedative antidepressants can improve the retention rate of sleep, and some antidepressants without a sedative effect yield the opposite effect. Therefore, the total amount of rest, the number of rest bouts, the length of the rest bouts, the total activity, and the waking activity were used to assess the diverse effects of the different prescriptions (Fig. 1C).

Ultraperformance liquid chromatography (UPLC) and quadrupole time-of-flight mass spectrometry (Q-TOF-MS) analyses were conducted for each prescription. The protonated $[\mathrm{M}+\mathrm{H}]^{+}$or deprotonated $[\mathrm{M}-\mathrm{H}]^{-}$ions were obtained with as much characteristic fragment information as possible to deduce the molecular and elemental compositions of every constituent. The inferred chemical structure was compared with published data and the natural product information reported. The electrospray ionisation-mass spectrometry (ESI-MS) spectra were acquired in both the negative and positive ion voltage modes for each prescription. Here, we only present the results of the 1st, 2nd, 3rd, and 5th prescriptions in the positive ion voltage mode (Fig. 1D). Using this method, for example, 29 compounds were identified in the 1st prescription, and detailed identification results are presented in the attached Supplementary Table S1 (Supplementary Figure S1).

Behavioural similarities induced by the same class of TCM prescriptions. We applied clustering algorithms to perform hierarchical clustering (Supplementary Figure S2) and k-means clustering according to the shared behaviour of the 7 prescriptions (Supplementary Figure S3). The hierarchical clustering revealed the behavioural diversity induced by the 7 prescriptions. K-means clustering was performed from $\mathrm{k}=4$ to $\mathrm{k}=10$. When the concentration was lower (less than $50 \mu \mathrm{g} / \mathrm{mL}$ ), the 5 parameters of the treated groups exhibited no significant differences compared with those of the controls. Therefore, based on the concentration effects and the correlation between the concentration pairs, we only selected some concentrations for further k-means clustering. These concentrations yielded an observed effect and, at the same time, correlated well with each other; they were generated from the correlation matrixes (Supplementary Figure S4). This highly correlated k-means clustering greatly reduced the clustering numbers. As intended, the clustering was performed using $\mathrm{k}$ values from 3 to 6 (Supplementary Figure S5), and the clustering result of $\mathrm{k}=4$ was analysed in detail (Fig. 2A).

The clustering analysis revealed that the 1 st and 3rd prescriptions induced different behavioural phenotypes. The 2 nd and 4 th prescriptions co-clustered into one category due to the similar behavioural changes caused by these prescriptions. The 5 th, 6 th, and 7 th prescriptions co-clustered into one class. The 7 prescriptions played a role in treating depression from four different angles in TCM therapy; similarly, they were also divided into the same four categories according to the larval behavioural profiles. These classification results, which were based on TCM and zebrafish behavioural science, were consistent; these findings also demonstrated that the automatic video-recording system was feasible for studying the TCM prescriptions by means of rest/wake behaviour in zebrafish.

To compare the induced behaviours during the day and night, a time series analysis of the average waking activity and the average rest was conducted (Supplementary Figure S6). We randomly selected a prescription from each of the above mentioned four categories and obtained fingerprints and line graphs as discussed below. For the 1st 
A
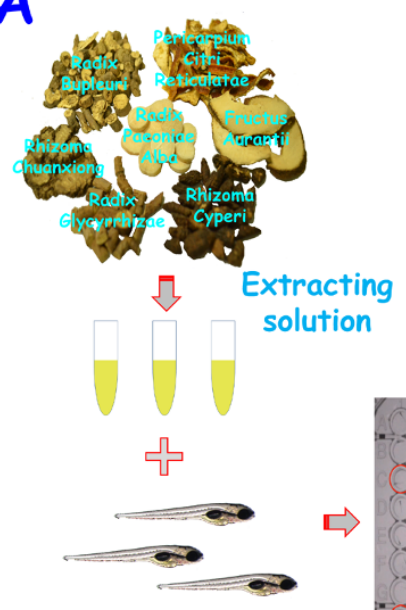

Larval zebrafish (4dpf)

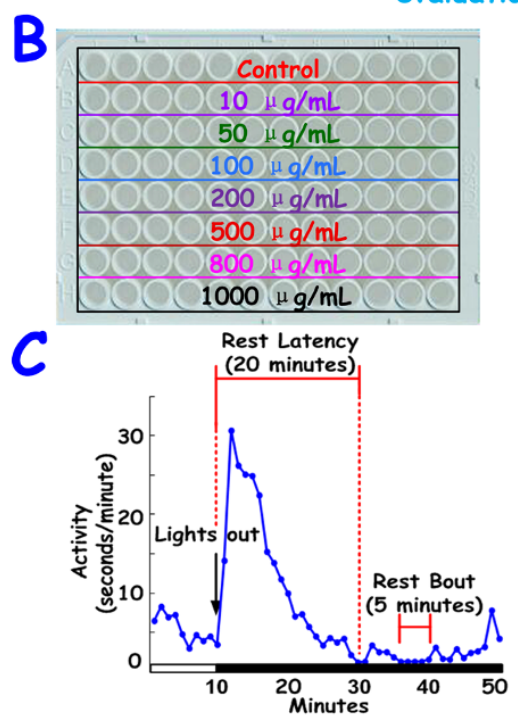

Behavioural analysis
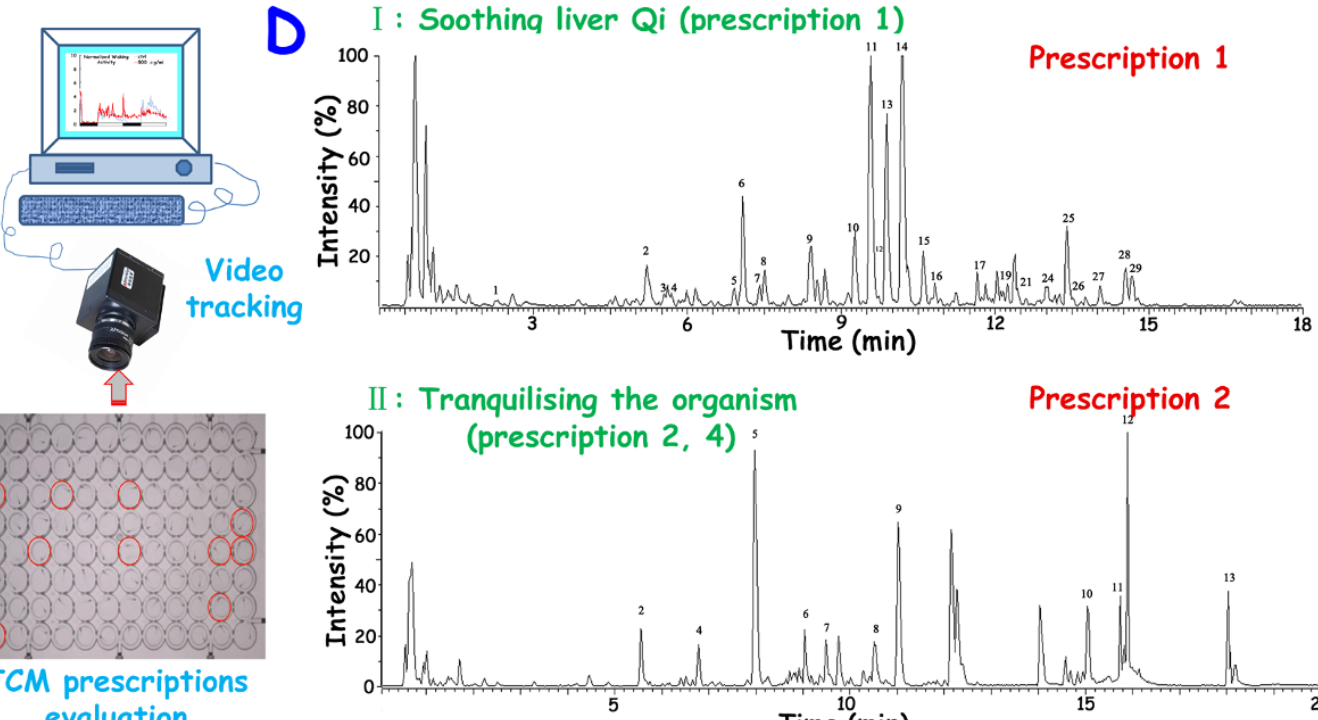

Prescription 2

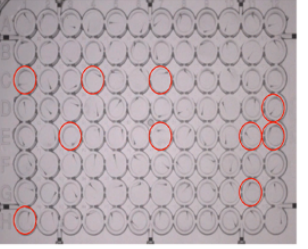

TCM prescriptions evaluation

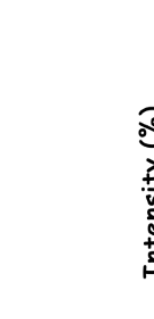

III: Soothing liver and activating spleen (prescription 3)

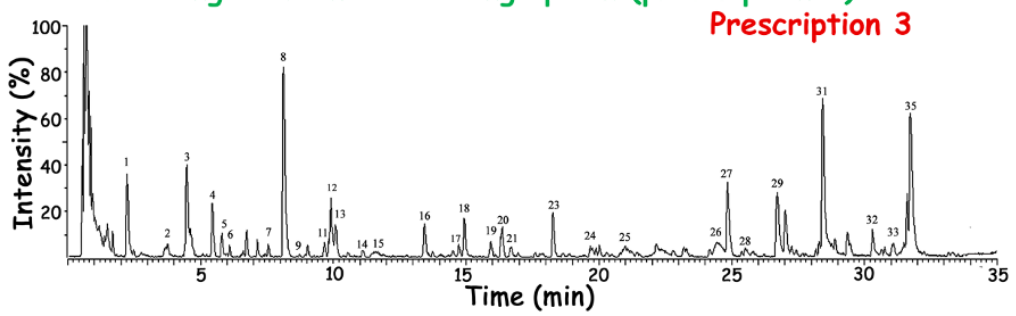

IV : Beina tonic (prescription 5, 6, 7)

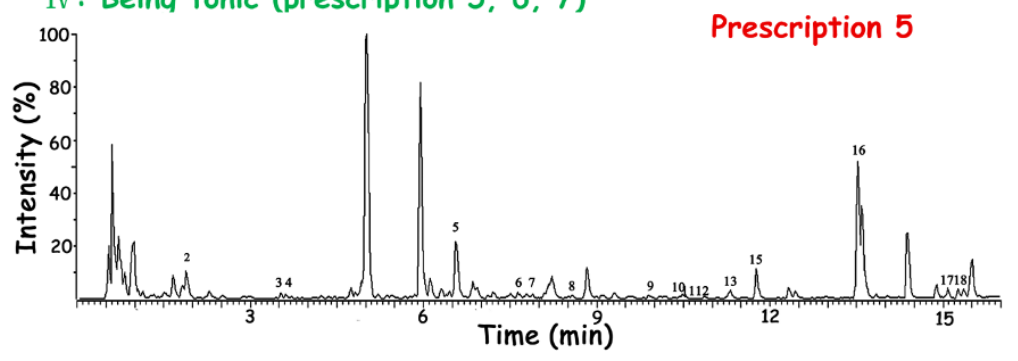

Figure 1 Rest/wake behavioural assay in larval zebrafish. (A) Crude herbs were prepared to obtain the extracting solution. Larvae (4 dpf) were then pipetted into each well of a 96-well plate $(\sim 360 \mu \mathrm{L})$ with different dilutions of TCM prescriptions at 9:00 AM. Zebrafish were observed beginning at 9:00 PM at 4 dpf. (B) As shown in this figure, the concentrations used were 10, 50, 100, 200, 500, 800, and $1000 \mu \mathrm{g} / \mathrm{mL}$. Twelve larvae were prepared for each group. Parallel experiments were performed at least three times for each prescription. (C) Locomotor activity of a representative larva. Five parameters, including the total amount of rest, the number of rest bouts, the length of the rest bouts, the total activity, and the waking activity, were analysed in detail. Each value represents the average of $\sim 30$ larvae, and these parameters then generated the behavioural fingerprint of each prescription. (D) ESI-MS spectra in the positive ion voltage mode of the 1st, 2nd, 3rd, and 5th prescriptions. Some of the constituents are labelled in the spectra.

prescription, wakefulness increased on the second night and decreased on the second day only. During the experimental period, the total rest was consistently decreased (Fig. 2B). The 2nd prescription was similar to the 4 th prescription; they induced a similar behavioural changes. The wakefulness was slightly increased at night, and the total amount of rest was decreased. However, a decrease in wakefulness occurred during the last period (Fig. 2C). The 3rd prescription selectively increased wakefulness and decreased the amount of rest during the day, and it had little effect at night (Fig. 2D). The 5th, 6 th, and 7 th prescriptions induced a similar behavioural change. The waking activity was decreased on the second day, and the total amount of rest did not change significantly throughout the experimental period (Fig. 2E).

Quantitative analysis of behavioural measurements caused by each TCM prescription. To compare the difference in each para- meter between the experimental groups and the controls more clearly, we analysed the behavioural fingerprints. In addition, the five parameters were quantitatively analysed, and the results are discussed below. Compared with the controls, the 1st prescription decreased the total amount of rest and the length of the rest bouts and increased the number of rest bouts and the total activity. At the doses of 500 and $800 \mu \mathrm{g} / \mathrm{mL}$, the total amount of rest and waking activity were significantly different from that of the controls (Fig. 3A). The 2nd prescription greatly reduced the total amount of rest and waking activity during the experimental period. Its curative effect was more intense, and the lowest observed effective concentration (LOEC) was $100 \mu \mathrm{g} / \mathrm{mL}$. As the concentrations became higher, the rest total and waking activity decreased; however, on the 6th day, the prescription caused a sharp rise in the rest total and the waking activity at a dose of $1000 \mu \mathrm{g} / \mathrm{mL}$ (Fig. 3B). The $3 \mathrm{rd}$ prescription had a small effect on the 5 parameters. At 50,100 , and $200 \mu \mathrm{g} / \mathrm{mL}$, the total amount of rest 


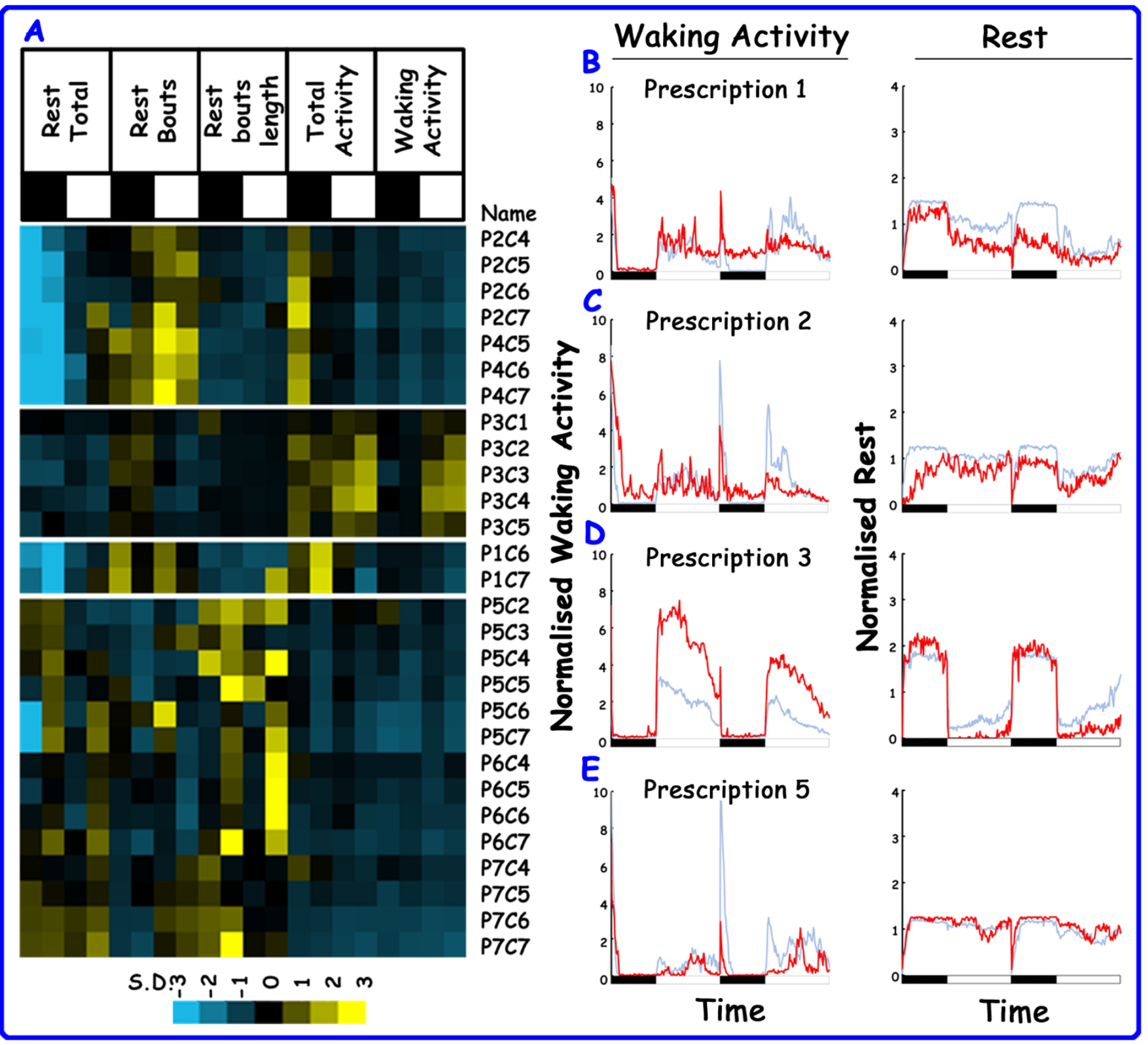

Figure 2 Clustering analysis revealed the behavioural similarities between the TCM prescriptions. (A) K-means clustering analysis. The clustergram was divided into four sectors, each of which represents a similar behavioural phenotype. Each row represents a unique concentration of a certain prescription, and each column indicates a behavioural measurement. From left to right, these measurements are the following: the total amount of rest, the number of rest bouts, the length of the rest bouts, the total activity, and the waking activity. The black bars indicate the night measurements, and the white bars indicate the day measurements. The measurements were normalised as standard deviations from the controls. The yellow and blue colours indicate that the values are higher and lower relative to controls, respectively. In this clustergram, "P" and "C" represent "prescription" and "concentration", respectively. For example, "P1C6" represents the 6th concentration $(800 \mu \mathrm{g} / \mathrm{mL})$ of the 1 st prescription. (B-E) The waking activity and the rest total were averaged in 10-minute intervals and then normalised to the control values. In the time series analysis, the red trace indicates the average of the treated group, and the blue trace indicates the average of the controls. The black and white bars represent the night and day measurements, respectively.

was decreased and the waking activity was increased. This prescription induced a greater effect during the day compared with the night (Fig. 3C). The 5th prescription dramatically decreased wakefulness and increased the length of the rest bouts. Interestingly, it increased the total rest amount during the first and second days. Its LOEC was $50 \mu \mathrm{g} / \mathrm{mL}$, and it caused a slight reduction in the total activity (Fig. 3D). The 4 th prescription was similar to the 2 nd prescription; it tended to cause a decrease in the total amount of rest, length of the rest bouts, total activity, and waking activity. The number of rest bouts was increased. At lower concentrations, it had little effect. The 6th prescription induced a slight decrease in the number of rest bouts, total activity, and waking activity. On the second day, the length of the rest bouts increased significantly. The 7th prescription caused an increase in the rest total and the number of rest bouts. It had a different effect on the number of rest bouts at night compared with that during the daytime. Wakefulness was considerably decreased (Supplementary Figures S7-8).

Prediction of the effect of TCM prescriptions on the neural signalling pathway. The correlation matrix was plotted to assess the similarities between all prescription pairs (Supplementary Figure S9). As the dendrogram indicates (Fig. 4), compared with 


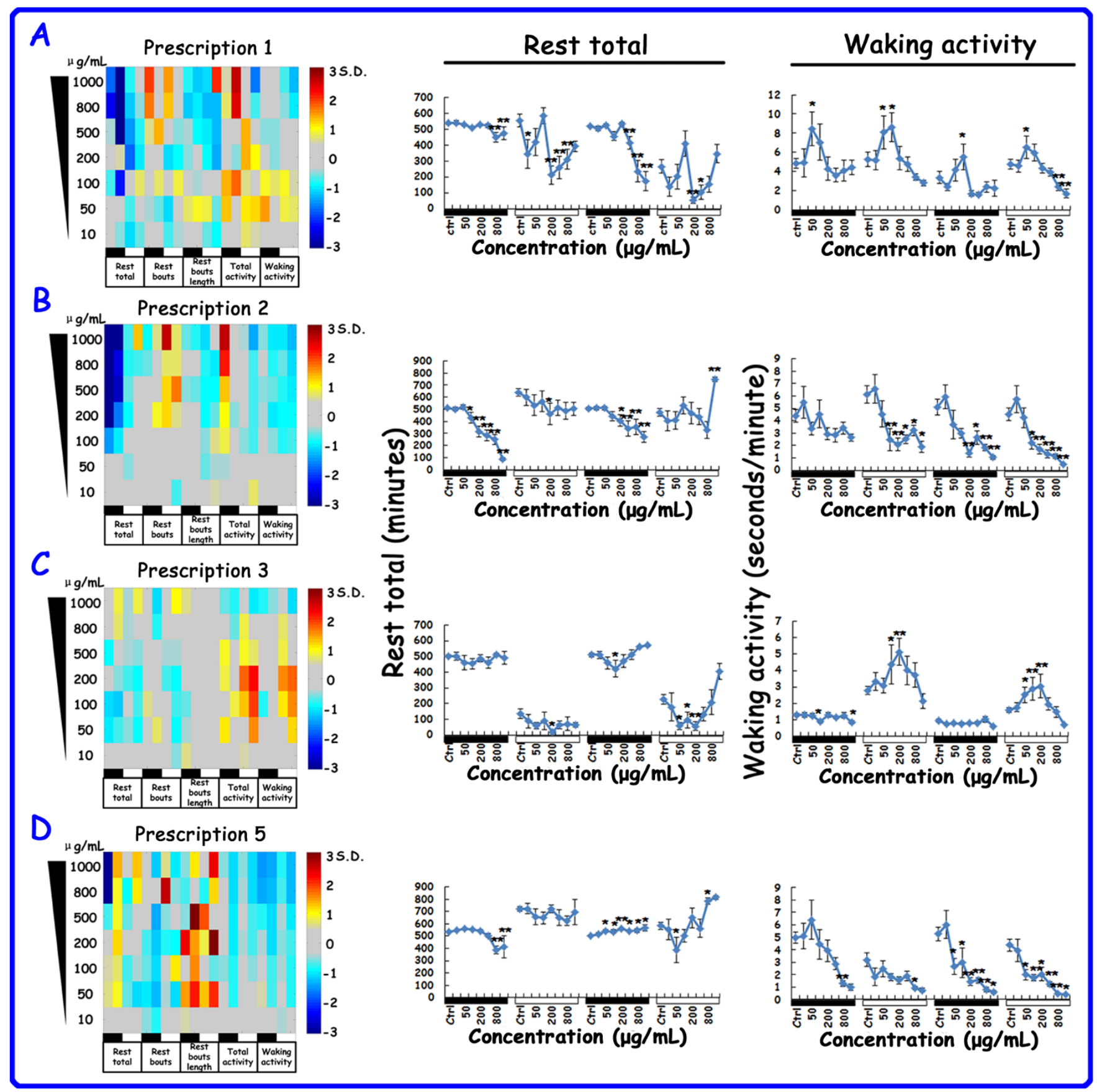

Figure 3| Qualitative and quantitative analyses of behavioural profiles induced by seven prescriptions. (A-D) In the left panel, the behavioural fingerprints for each dose were calculated as \pm the standard deviations from the controls. In these fingerprints, the red and blue colours indicate higher and lower values, respectively, relative to the controls. The black and white bars indicate the night and day measurements, respectively. From left to right, these parameters are the total amount of rest, number of rest bouts, length of the rest bouts, total activity, and waking activity. The middle and right panels show the line graphs that were generated to analyse the total amount of rest and waking activity in detail. Error bars represent the standard error of the means (SEM). In our study, $\mathrm{P}<0.05\left(^{*}\right)$ and $\mathrm{P}<0.01\left({ }^{*}\right)$ were considered to be significantly different relative to the controls. Each value represents the average of $\sim 30$ larvae (Because of natural or drug factors, larvae had different degrees of death.) (Supplementary Figure S12). The black and white bars indicate the night and day measurements, respectively.

other prescriptions, the 1st prescription had a moderate correlation with the 2nd and 4 th prescriptions. Furthermore, the 2nd and 4th prescriptions were more similar. The 3rd prescription did not correlate well with any prescription. The 5th, 6th, and 7th prescriptions were highly correlated with each other.

To reveal the fundamental mechanisms associated with the treatment of depression among the 7 prescriptions, we selected approximately 500 compounds for the correlation analysis (Supplementary
Figure S10). The compounds were obtained from Schier's report. In their report, a high-throughput method was used to screen hundreds of psychoactive compounds and predicted the biological targets of some poorly characterised compounds ${ }^{44}$. In their hierarchical diagram, different colours represent different parameter values. We calculated the parameters from the colours. The colour value (Red, Green, Blue $[\mathrm{RGB}])$ was $(0,0,0)$ for the parameter of 0 in the colour bar. We set the RGB values of parameters 3 (the yellow block on the 


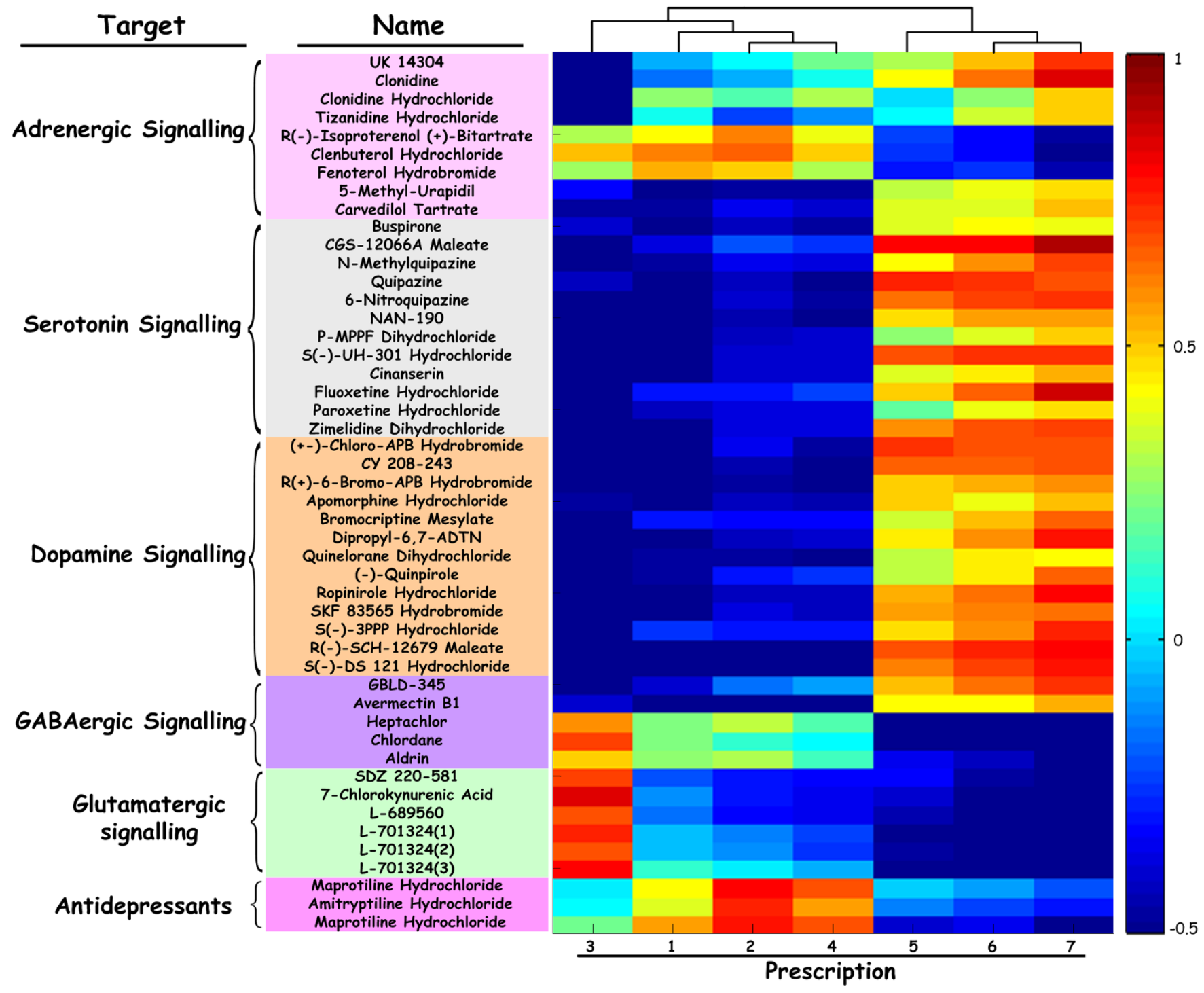

Figure 4 Correlation analysis of the 7 TCM prescriptions and known compounds. The weighted uncentred Pearson's correlation coefficient between each compound and each prescription was calculated. The X axis denotes the 7 TCM prescriptions, and the Y axis denotes the compounds. The upper dendrogram was conducted with hierarchical clustering. In the left, the chemical names are coloured according to the activity code annotations. For example, serotonin signalling is labelled grey. Compounds associated with different neural pathways are labelled in different colours. However, there is an exception in the last colour block, which includes two classical antidepressants that did not belong to a neural signalling pathway. In the colour bar, red indicates a high correlation, and blue indicates a low correlation.

right) and -3 (the blue block on the left) to $\left(R_{y}, G_{y}, B_{y}\right)$ and $\left(R_{b}, G_{b}\right.$, $\left.B_{b}\right)$, respectively, in the colour bar. If the RGB value of the colour block was $(r, g, b)$ in the dendrogram, the corresponding parameter could be calculated by the following equation:

$$
\text { para }=\left\{\begin{array}{cc}
1.5 *\left(\frac{r}{R_{y}}+\frac{g}{G_{y}}\right) & B_{y}=0 \\
-1 *\left(\frac{r}{R_{b}}+\frac{g}{G_{b}}+\frac{b}{G_{b}}\right) & B_{y} \neq 0
\end{array}\right.
$$

Based on the correlation analysis of the 7 TCM prescriptions and $\sim 500$ compounds, we found that the TCM prescriptions had a high correlation with some compounds with known biological targets. The analysis indicated that the 5 th, 6 th, and 7 th prescriptions were likely to be related to the adrenergic, serotonin (5-HT), and dopamine (DA) signalling pathways, respectively. 5-HT, norepinephrine (NE), and DA are monoamine neurotransmitters, which have extensive biological activities and are involved in many physiological responses of the central nervous system, such as emotional responses, mental activity, thermoregulation, and sleep. To date, most studies have shown that depression is associated with abnormal neurotransmitter metabolism ${ }^{45}$. A study has also demonstrated that 5-HT, NE, and DA contents in the hypothalamus and hippocampus were significantly decreased in depressed rats compared with the normal groups ${ }^{46}$. To date, monoamine theory is one of the most studied areas in the depression literature $e^{47-49}$. It has been demonstrated that a decreased level/function of monoamine neurotransmitters in the synaptic cleft may be the biological basis of depression ${ }^{50}$.

The 3rd prescription may be associated with the GABAergic $(\gamma-$ amino butyric acid) and glutamatergic signalling pathways. GABA is an inhibitory neurotransmitter in the central neural system and plays a prominent role in many physiological and psychological processes $^{51}$. Dysfunction of the GABAergic system has been shown to have substantial interactions with some psychiatric disorders, including major depression ${ }^{52,53}$. Glutamate is an excitatory neurotransmitter in mammals, and it plays important roles in the pathophysiology and treatment of major depressive disorder (MDD) ${ }^{54,55}$. 
Abnormalities in glutamate cycling in the brain have been found in patients of $\mathrm{MDD}^{56}$.

In addition, we found that the $2 \mathrm{nd}$ and 4 th prescriptions yielded a behavioural phenotype similar to that of two classical antidepressants, amitriptyline and maprotiline. Amitriptyline is a tricyclic antidepressant, and maprotilineis is a tetracyclic antidepressant. By blocking monoamine neurotransmitter reuptake and increasing the levels of monoamines in the synaptic cleft, they can improve the depressed mood. The two prescriptions may have a similar mechanism of action in depression.

\section{Discussion}

Depression is a complex disease that involves hereditary, biochemical, psychological, social-environmental, and other factors. From the standpoints of neurophysiology, neuroanatomy, and neuropathology, most scholars predict that the underlying cause of depression is a decreased level of neurotransmitters. However, the study of depression is far from complete. Some physical and chemical analyses are limited to experimental study and cannot be used in the clinic. Current antidepressants can improve depressive symptoms but cannot prevent the recurrence of the disorder. Most antidepressants have significant side effects, which significantly influence patients' lives. Therefore, combining TCM and modern medicine is necessary.

The clinical manifestations of depression vary. As previously described, TCM posits that depression is involved in multiple organs. Of greater importance is the characterisation of TCM prescriptions by multiple constituents, multiple targets, and multiple pathways. Therefore, this study possesses significant potential to aid the development of depression treatments. TCM focuses on the overall effect of medicine, and it also plays a role in health care and disease prevention through the treatment of multiple targets. It can yield stable and comprehensive curative effects and greatly reduce the side effects of drugs. In our study, the video-tracking system yielded highly accurate results (Supplementary Video S1), and the zebrafish rest/wake assay indicated that antidepressant prescriptions had a good curative effect. Furthermore, we predicted the mode of action of poorly characterised prescriptions. However, all 7 prescriptions contained a variety of single herbs, and each individual herb contained a variety of ingredients. The constituent(s) that played a leading role in the treatment remains unknown. By analysing the chemical components of seven prescriptions, we found that some active components existed in TCM prescriptions. For example, the 5th, 6th, and 7th prescriptions contained several important polyphenols and ginsenoside, including ferulic acid, naringenin, nobiletin, hesperidin, chlorogenic acid, and apigenin (Supplementary Figure S11). Studies have shown that these polyphenols and ginsenoside possessed antidepressant-like activity in animal model of depres$\operatorname{sion}^{57-60}$. We will further screen the active components of each TCM prescription using the larval locomotor activity assay, thereby isolate them from the mixtures of prescriptions. Whether the mixtures are needed for activity requires further research. Meanwhile, use of other behavioural assays is also being considered to round out the mechanism of action of TCM prescriptions. By extracting the active components and producing a high-efficiency TCM preparation, we hope to benefit more patients with depression and facilitate the process of internationalisation of TCM.

\section{Methods}

Preparation, UPLC analysis, and Q-TOF-MS analysis of the prescriptions. The composition of Chai-Hu-Shu-Gan-San (prescription 1) was as follows (the values indicate the proportions compared with those of the other ingredients): Radix Bupleuri 4, Pericarpium Citri Reticulatae 4, Radix Paeoniae Alba 3, Radix Glycyrrhizae 1, Fructus Aurantii 3, Rhizoma Chuanxiong 3, and Rhizoma Cyperi 3. Gan-Mai-Da-Zao-Tang (prescription 2) was composed of Radix Glycyrrhizae 2, Triticum Aestivum 3, and Fructus Jujubae 3. Gui-Pi-Tang (prescription 3) was composed of Rhizoma Atractylodis Macrocephalae 2, Radix Angelicae Sinensis 2, Poria 2, Radix Astragali 2, Cortex et Radix Polygalae 2, Arillus Longan 2, Semen
Ziziphi Spinosae 2, Radix Ginseng 4, Radix Auck landiae 1, and Radix Glycyrrhizae 1. The composition of Wen-Dan-Tang (prescription 4) was Rhizoma Pinelliae 4, Caulis Bambusae in Taeniam 4, Fructus Aurantii Immaturus 4, Pericarpium Citri Reticulatae 6, Radix Glycyrrhizae 2, and Poria 3. Tao-Hong-Si-Wu-Tang (prescription 5) was composed of equal proportions of Semen Persicae, Flos Carthami, Radix Angelicae Sinensis, Radix Rehmanniae Preparata, Rhizoma Chuanxiong, and Radix Paeoniae Alba. The composition of Qi-Ju-Di-Huang-Wan (prescription 6) was Fructus Lycii 1, Flos Chrysanthemi 1, Radix Rehmanniae Preparata 4, Fructus Corni 2, Cortex Moutan 1.5, Rhizoma Diosscoreae 2, Poria 1.5, and Rhizoma Alismatis 1.5. Liu-Jun-Zi-Tang (prescription 7) was composed of equal proportions of Radix Ginseng, Radix Glycyrrhizae, Poria, Rhizoma Atractylodis Macrocephalae, Pericarpium Citri Reticulatae, and Rhizoma Pinelliae.

All crude herbs were purchased from Changan Chinese Medicinal Materials Co., LTD (Anguo, Hebei, China) and identified by Prof. Tie-jun Zhang (Modern Research of Traditional Chinese Medicine Department, Tianjin Institute of Pharmaceutical Research, Tianjin, P. R. China). After approximately $100 \mathrm{~g}$ of each prescription was boiled gently in 10 times their volume of water for $60 \mathrm{~min}$, the obtained supernatant separated by filtration was evaporated under a vacuum and lyophilised by quick freezing to obtain the dry extract. The obtained sample was dissolved in $\mathrm{CH}_{3} \mathrm{CN}$ and filtered through a $0.2-\mu \mathrm{m}$ filter prior to UPLC analysis.

A Waters Acquity UPLC System (Waters, MA, USA) equipped with a photodiode array detector was used. UV detection was achieved in the range of 190 to $400 \mathrm{~nm}$. The system was controlled by Masslynx V4.1 software (Waters Co.). An acquity BEH C18 column $(2.1 \times 100 \mathrm{~mm}, 1.7 \mu \mathrm{m}$; Waters Co. $)$ was used for the separations. Using Chai-Hu-Shu-Gan-San as an example, a gradient elution of $0.1 \%$ formic water (A) and acetonitrile (B) was performed as follows: $2 \% \mathrm{~B}$ was obtained from 0 to $2 \mathrm{~min}, 2$ to $23 \%$ B from 2 to $10 \mathrm{~min}, 23$ to $38 \%$ B from 10 to $12 \mathrm{~min}, 38$ to $55 \%$ B from 12 to $17 \mathrm{~min}$, and 55 to $100 \% \mathrm{~B}$ from 17 to $18 \mathrm{~min} ; 100 \% \mathrm{~B}$ was maintained from 18 to $20 \mathrm{~min} ; 100$ to $2 \% \mathrm{~B}$ was obtained from 20 to $21 \mathrm{~min}$; and $2 \% \mathrm{~B}$ was maintained from 21 to $23 \mathrm{~min}$. Other samples were slightly adjusted according to their ingredients and chemical polarity. The flow rate was $0.40 \mathrm{~mL} / \mathrm{min}$, and the column temperature was maintained at $40^{\circ} \mathrm{C}$.

Accurate mass measurements and MS/MS were performed on a Waters Q-TOF Premier with an electrospray ionisation (ESI) system (Synapt G2-S HDMS, Waters MS Technologies, Manchester, UK). The ESI-MS spectra were acquired in both the negative and positive ion voltage modes. The capillary voltages were set to $2.5 \mathrm{kV}$ for the negative mode and $3.0 \mathrm{kV}$ for the positive mode. The sample cone voltage was set to $30 \mathrm{~V}$. High-purity nitrogen was used as the nebulisation and auxiliary gas. The nebulisation gas was set at a flow rate of $700 \mathrm{~L} / \mathrm{h}$ at $400^{\circ} \mathrm{C}$, the cone gas was set a flow rate of $50 \mathrm{~L} / \mathrm{h}$, and the source temperature was $120^{\circ} \mathrm{C}$. The Q-TOF Premier acquisition rate was $0.1 \mathrm{~s}$, with a 0.02 -s scan delay. The instrument was operated with the first resolving quadrupole in a wide pass mode (50 to 2,500 Da) and with the collision cell operating at two alternative energies (i.e., 5 and $30 \mathrm{eV}$ ). Leucine Enkephalin $(200 \mathrm{pg} / \mu \mathrm{L})$ was used as the lock mass $\left([\mathrm{M}-\mathrm{H}]^{-} 554.2615,[\mathrm{M}+\mathrm{H}]^{+}\right.$ 556.2771).

Zebrafish. The zebrafish were raised at a constant temperature of $28.5^{\circ} \mathrm{C}$ on a constant light cycle (14 h light/10 h dark). The fish-farming system was equipped with circulating water. To maintain a suitable salinity, the $\mathrm{pH}$ value, and the balance of electrolytes, $10 \%$ of the fresh deionised water was replenished daily. The zebrafish were fed frozen brine shrimp three times daily. One male and one female adult zebrafish were introduced to a single breeding tank with a sliding door in advance. The next morning, the sliding door was removed to allow the male to pursue the female. The fertilised eggs were then collected after one hour and transferred to a clean flat dish with standard egg water $\left(\mathrm{KCl} 0.05 \mathrm{~g} / \mathrm{L}, \mathrm{NaHCO}_{3} 0.025 \mathrm{~g} / \mathrm{L}, \mathrm{NaCl} 3.5\right.$ $\mathrm{g} / \mathrm{L}$, and $\mathrm{CaCl}_{2} 0.1 \mathrm{~g} / \mathrm{L}$, with $1 \mathrm{mg} / \mathrm{L}$ methylene blue, $\mathrm{pH}$ 7.0). The dead embryos were removed from the culture dish and all solutions were replaced at least twice daily. The embryos and larvae were raised until 4 days post-fertilisation (dpf), and the behavioural assay was then initiated at 9:00 PM at $4 \mathrm{dpf}$. All experimental protocols and procedures involving zebrafish were approved by the Committee for Animal Experimentation of the College of Life Science at Nankai University (no. 2008) and were performed in accordance with the NIH Guide for the Care and Use of Laboratory Animals (no. 8023, revised in 1996).

Exposure to the TCM extracting solution. To determine the effects of different dosages of the TCM prescriptions on larvae in vivo, we used a range of concentrations $(10,50,100,200,500,800$, and $1000 \mu \mathrm{g} / \mathrm{mL})$. First, the standard tank water was added to the dry extract to obtain the stock solution of $36 \mathrm{mg} / \mathrm{mL}$. Prior to the exposure to these TCM prescriptions, the larval zebrafish were transferred to each well of a 96well plate. A total of $360 \mu \mathrm{L}$ of different dilutions $(10,50,100,200,500,800$, and $1000 \mu \mathrm{g} / \mathrm{mL}$ ) of TCM was pipetted into each well containing the larva, and standard tank water was added to a separate well as a control. Twelve larvae were tested at each concentration. To minimise evaporation, the 96-well plate was put into a transparent glass box and sealed with a layer of plastic film.

Video-tracking system. To access the larvae's rest/wake behaviour, an imaging system was introduced. A 96-well plate was placed on a transparent acrylic shelf to hold the larvae. An infrared LED array, with an acrylic diffuser located above it, was utilised as a back light source. A camera (MV-VS078FM, Microvision), which was fitted with a fixed-angel megapixel lens (MP5018, Computar) and an infrared filter, was fixed on a crane for vertical observation of the larvae. To obtain the larvae's rest/ wake behavioural data, a custom program was developed with Microsoft Visual 
Studio 2010 and OpenCV 2.4.3. The program captured high-quality images of the zebrafish larvae (with a resolution of $1024^{*} 768$ ) at $10 \mathrm{fps}$ and recorded the time series of each larva's rest/wake behaviour. An algorithm based on the subtraction of the adjacent frame was applied; this algorithm was reliable because the back light source yielded distinct images (Supplementary Video S1).

In our experiment, all equipment was maintained in a room with illumination from 7:00 AM to 9:00 PM and at a constant temperature of $28.5^{\circ} \mathrm{C}$. The larvae's rest/wake behavioural data over 48 consecutive hours were recorded beginning at 9:00 PM at 4 dpf.

Data analysis. The raw data for each zebrafish larva's rest/wake behaviour, which were obtained from our imaging system, consisted of a time series. Each number in the time series was the number of movements of a larva in each minute during 48 consecutive hours. Because the camera captured each frame every 0.1 seconds, the raw behavioural data of each larva also indicated the amount of time for which the larva had moved. Our method was based on parameters that were manually defined and characterised the larva's rest/wake regulation. The 5 parameters, including the total amount of rest, the number of rest bouts, the length of the rest bouts, the total activity, and the waking activity, for each day and night were first extracted from the raw time series. Every minute with less than 0.3 seconds of movement was defined as a rest minute. A rest bout was defined as a continuous series of rest minutes. The parameters were defined as follows:

1. Rest total: the number of rest minutes in a single day/night

2. Number of rest bouts: the number of rest bouts in a single day/night.

3. Length of the rest bouts: the average length of a continuous series of rest minutes.

4. Total activity: the average duration of movements in a single day/night.

5. Waking activity: the average duration of movements in a single day/night excluding all rest minutes.

Twelve larvae were tested at each concentration, and the experiment for each prescription was replicated three times. The data for each parameter in each period were the average of three experiments. To identify the TCM prescriptions that significantly altered the locomotor behaviour, each parameter in the treated groups was compared with that of the controls using one-way ANOVA. The data were expressed as the means \pm standard error of the mean (SEM). $\mathrm{P}<0.05$ was considered to indicate a significant difference. Differences between the experimental groups and the controls were tested for significance with SPSS 19.0, and the line graphs were obtained with Excel 2010.

Each observation lasted for 2 days and 2 nights; thus, 20 parameters were extracted. For further analysis, to avoid domination of the attributes with greater values over those with smaller values, the 20 parameters were then normalised to the controls and constituted a feature vector. Seven concentrations of each of the 7 prescriptions were tested, which led to 49 feature vectors. K-means clustering and hierarchical clustering were performed with Cluster 3.0 and Java Tree View, and all clustergrams were plotted with Microsoft Visio. K-means clustering was performed based on the Euclidean distance. For each prescription, the feature vectors of the phenotypes at different dosages were calculated and are shown as behavioural fingerprints. To compare the differences between the different dosages, the uncentred Pearson correlations of the feature vectors for each prescription were calculated and are presented in a correlation matrix. In each time series, the total amount of rest and the waking activity were averaged across $\sim 30$ larvae in 10-minute intervals, and they were then normalised to the controls. The controls were plotted for comparison. The behavioural fingerprint, correlation matrix, and time series were visualised with Matlab R2011b (MathWorks).

1. Belmaker, R. H. \& Agam, G. Major depresssive disorder. N. Engl. J. Med. 358, 55-68 (2008).

2. Richelson, E. Pharmacology of antidepressants. Mayo Clin. Proc. 76, 511-527 (2001).

3. Perrine, S. A. et al. Ketamine reverses stress-induced depression-like behaviour and increased GABA levels in the anterior cingulate: An 11.7 T ${ }^{1} \mathrm{H}$-MRS study in rats. Prog. Neuro-Psychopharmacol. Biol. Psychiatry. 51, 9-15 (2013).

4. Bauer, M., Tharmanathan, P., Volz, H. P., Moeller, H. J. \& Freemantle, N. The effect of venlafaxine compared with other antidepressants and placebo in the treatment of major depression: a meta-analysis. Eur. Arch. Psychiatry. Clin. Neurosci. 259, 172-185 (2009).

5. Licinio, J. \& Wong, M. L. Depression, antidepressants and suicidality: a critical appraisal. Nat. Rev. Drug Discov. 4, 165-171 (2005).

6. Halbreich, U. Major depression is not a diagnosis, it is a departure point to differential diagnosis-clinical and hormonal considerations: (a commentary and elaboration on Antonejevic's paper). Psychoneuroendocrinology. 31, 16-22 (2006).

7. Blais, R. K. \& Renshaw, K. D. The association of biological and psychological attributions for depression with social support seeking intentions in individuals with depressive symptoms. Behav Cogn Psychother. 40, 605-617 (2012).

8. Ong, K. S. \& Keng, S. B. The biological, social and psychological relationship between depression and chronic pain. Cranio. 21, 286-294 (2003).

9. Wilson, M. P., Castillo, E. M., Batey, A. M., Sapyta, J. \& Aronson, S. Hepatitis C and depressive symptoms: psychological and social factors matter more than liver injury. Int. J. Psychiatr. Med. 40, 199-215 (2010).
10. Antunes, P. B. \& Fleck, M. P. Clinical outcomes and quality of life in patients submitted to electroconvulsive therapy. J. ECT. 25, 182-185 (2009).

11. Wijeratne, C. \& Sachdev, P. Treatment-resistant depression: critique of current approaches. Aust N Z J Psychiatry. 42, 751-762 (2008).

12. Belmaker, R. H. The future of depression psychopharmacology. CNS Spectr. 13, 682-7 (2008).

13. Tokita, K., Yamaji, T. \& Hashimoto, K. Roles of glutamate signaling in preclinical and/or mechanistic models of depression. Pharmacol., Biochem. Behav. 100, 688-704 (2012).

14. Racagni, G. \& Popoli, M. The pharmacological properties of antidepressants. Int. Clin. Psychopharmacol. 25, 117-31 (2010).

15. Anderson, H. D., Pace, W. D., Libby, A. M., West, D. R. \& Valuck, R. J. Rates of 5 common antidepressant side effects among new adult and adolescent cases of depression: A retrospective US claims study. Clin. Ther. 34, 113-123 (2012).

16. Rascati, K., Godley, P. \& Pham, H. Evaluation of resources used to treat adverse events of selective serotonin reuptake inhibitor use. Manag Care Pharm. 7, 402-406 (2001)

17. Bull, S. A. et al. Discontinuing or switching selective serotoninreup take inhibitors. Ann. Pharmacother. 36, 578-584 (2002).

18. Taylor, D. Antidepressant drugs and cardiovascular pathology: a clinical overview of effectiveness and safety. Acta Psychiatr. Scand. 118, 434-442 (2008).

19. George, M. \& Burns Ewald, M. Three percent hypertonic saline as a therapy in reversal of the cardio toxic effects induced by tricyclic antidepressants. Clin. Toxicol. 46, 368-368 (2008).

20. Acikalin, A. et al. QTc intervals in drug poisoning patients with tricyclic antidepressants and selective serotonin reuptake inhibitors. Am. J. Ther. 17, 30-33 (2010)

21. Scheid, V. Depression, constraint, and the liver: (Dis) assembling the treatment of emotion-related disorders in Chinese medicine. Cult Med Psychiatry. 37, 30-58 (2013).

22. Ng, S. M., Chan, C. L. W., Ho, D. Y. F., Wong, Y. \& Ho, R. T. H. Stagnation as a distinct clinical syndrome: comparing "Yu" (stagnation) in traditional Chinese medicine with depression. BJSW. 36, 467-484 (2006).

23. Katon, W. J. Epidemiology and treatment of depression in patients with chronic medical illness. Dialogues Clin Neurosci. 13, 7-23 (2011).

24. Spijkerman, T. et al. Depression following myocardial infarction: first-ever versus on going and recurrent episodes. Gen. Hosp. Psychiatry. 27, 411-417 (2005).

25. Du, Y. H. \& Li, G. P. Theoretical basis of regulating mind and relieving the depressed liver needling method for treatment of depression. CAM. 25, 143-6 (2005).

26. Li, X. H., Li, J. J., Liu, Y. Y. \& Chen, J. X. Central neurobiological mechanism of liver depression and spleen deficiency syndrome based on chronic stress: a review. JCIM. 10, 1-6 (2012).

27. Bianchi, G. et al. Psychological status and depression in patients with liver irrhosis. Dig. Liver Dis. 37, 593-600 (2005)

28. Kim, S. H. et al. Antidepression effect of Chaihu-Shugan-San extract and its constituents in rat models of depression. Life Sci. 76, 1297-1306 (2005).

29. Wang, Y., Fan, R. \& Huang, X. Meta-analysis of the clinical effectiveness of traditional Chinese medicine formula Chaihu-Shugan-San in depression. J. Ethnopharmacol. 141, 571-577 (2012).

30. Zhang, Y. J. et al. Ferulic acid-induced anti-depression and prokinetics similar to Chaihu-Shugan-San via polypharmacology. Brain Res. Bull. 86, 222-228 (2011).

31. Zhang, G. \& Ruan, J. Treatment of mental depression due to liver-qi stagnancy with herbal decoction and by magnetic therapy at the acupoints-a report of 45 cases. J. Tradit. Chin. Med. 24, 20-1 (2004).

32. Niv, N. et al. The use of herbal medications and dietary supplements by people with mental illness. Community Ment Health J. 46, 563-569 (2010).

33. Sarris, J., Kavanagh, D. J. \& Byrne, G. Adjuvant use of nutritional and herbal medicines with antidepressants, mood stabilizers and benzodiazepines. J. Psychiatr. Res. 44, 32-41 (2010).

34. Kou, M. J. \& Chen, J. X. Integrated traditional and Western medicine for treatment of depression based on syndrome differentiation: a meta-analysis of randomized controlled trials based on the Hamilton depression scale. J. Tradit. Chin. Med. 32, $1-5$ (2012).

35. Kishimoto, N., Shimizu, K. \& Sawamoto, K. Neuronal regeneration in a zebrafish model of adult brain injury. Dis. Model. Mech. 5, 200-209 (2012).

36. Maximino, C., de Brito, T. M., de Mattos Dias, C. A. G., Gouveia, A. Jr. \& Morato, S. Scototaxis as anxiety-like behaviour in fish. Nat. Protoc. 5, 209-216 (2010).

37. Norton, W. \& Bally-Cuif, L. Adult zebrafish as a model organism for behavioural genetics. BMC Neurosci. 11, 90 (2010).

38. Allgood, O. E. Jr. et al. Estrogen prevents cardiac and vascular failure in the 'listless' zebrafish (Daniorerio) developmental model. Gen. Comp. Endocrinol. 189, 33-42 (2013).

39. Kokel, D. et al. Rapid behaviour-based identification of neuroactive small molecules in the zebrafish. Nat. Chem. Biol. 6, 231-237 (2010).

40. Cachat, J. et al. Measuring behavioural and endocrain responses to novelty stress in adult zebrafish. Nat. Protoc. 5, 1786-1799 (2010).

41. Egan, R. J. et al. Understanding behavioural and physiological phenotypes of stress and anxiety in zebrafish. Behav. Brain Res. 205, 38-44 (2009).

42. Sison, M. \& Gerlai, R. Associative learning in zebrafish (Daniorerio) in the plus maze. Behav. Brain Res. 207, 99-104 (2010). 
43. Padilla, S., Hunter, D. L., Padons, B., Frady, S. \& MacPhail, R. C. Assessing locomotor activity in larval zebrafish: Influence of extrinsic and intrinsic variables. Neurotoxicol. Teratol. 33, 624-630 (2011).

44. Rihel, J. et al. Zebrafish behavioural profiling links drugs to biological targets and rest/wake regulation. Science. 327, 348-351 (2010).

45. Moret, C. \& Briley, M. The importance of norepinephrine in depression. Neuropsychiatr. Dis. Treat. 7, 9-13 (2011).

46. Xu, S. F., Zhuang, L. X., Tang, C. Z. \& Yang, J. J. Effects of acupuncture and embedding thread on central monoamine neurotransmitters in the depression model rat. CAM. 27, 435-437 (2007).

47. Hindmarch, I. Expanding the horizons of depression: beyond the monoamine hypothesis. Hum. Psychopharmacol. 16, 203-218 (2001).

48. Prins, J., Olivier, B. \& Korte, S. M. Triple reuptake inhibitors for treating subtypes of major depressive disorder: the monoamine hypothesis revisited. Expert Opin. Investig. Drugs. 20, 1107-1130 (2011).

49. Shulman, K. I., Herrmann, N. \& Walker, S. E. Current place of monoamine oxidase inhibitors in the treatment of depression. CNS Drugs. 27, 789-797 (2013).

50. Delgado, P. L. Depression: The case for a monoamine deficiency. J. Clin. Psychiatry. 61, 7-11 (2000).

51. Slattery, D. A. \& Cryan, J. F. The role of $\mathrm{GABA}_{\mathrm{B}}$ receptors in depression and antidepressant-related behavioural responses. Drug Dev. Res. 67, 477-494 (2006).

52. Slattery, D. A. \& Cryan, J. F. The role of GABA(B) receptors in depression and antidepressant-related behavioural responses. Drug Dev. Res. 67, 477-494 (2006).

53. Cryan, J. F. \& Slattery, D. A. GABA(B) receptors and depression: current status. Adv. Pharmacol. 58, 427-451 (2010).

54. Hashimoto, K. The role of glutamate on the action of antidepressants. Prog. Neuro-Psychopharmacol. Biol. Psychiatry. 35, 1558-68 (2011).

55. Zarate, C. Jr. et al. Glutamatergic modulators: the future of treating mood disorder? Harv. Rev. Psychiatr. 18, 293-303 (2010)

56. Valentine, G. W. \& Sanacora, G. Targeting glial physiology and glutamate cycling in the treatment of depression. Biochem Pharmacol. 78, 431-9 (2009).

57. Pathak, L., Agrawal, Y. \& Dhir, A. Natural polyphenols in the management of major depression. Expert Opin. Investig. Drugs. 22, 863-80 (2013)

58. Donato, F. et al. Hesperidin exerts antidepressant-like effects in acute and chronic treatments in mice: Possible role of I-arginine-NO-cGMP pathway and BDNF levels. Brain Res. Bull. 104, 19-26 (2014).

59. Lee, B., Shim, I., Lee, H. \& Hahm, D. H. Effect of ginsenoside Re on depressionand anxiety-like behaviors and cognition memory deficit induced by repeated immobilization in rats. J. Microbiol. Biotechnol. 22, 708-20 (2012).

60. Yamada, N., Araki, H. \& Yoshimura, H. Identification of antidepressant-like ingredients in ginseng root (Panax ginseng C. A. Meyer) using a menopausal depressive-like state in female mice: participation of $5-\mathrm{HT}_{2 \mathrm{~A}}$ receptors. Psychopharmacology. 216, 589-599 (2011).

\section{Acknowledgments}

This project was initiated in the State Key Laboratory of Medicinal Chemical Biology at Nankai University. We thank our colleagues for their detailed instructions and critical reading of the manuscript. Our work received financial support from the National Natural Science Foundation of China (grant no: 81373506 and 81071260), the Special Fund for Basic Research on Scientific Instruments of the Chinese National Natural Science Foundation (grant no: 61327802), and the State Key Laboratory of Medicinal Chemical Biology (grant no: 20130301).

\section{Author contributions}

X.Z.F. and G.B. conceived the idea for the study. X.Z.F. conceived and designed the experiment. Y.N.W. conducted the zebrafish behavioural assay and wrote the manuscript. The video-tracking software was designed by M.Z.S. and C.Y.Z., G.B. and Y.Y.H. supplied the TCM materials and provided suggestions. X.Z. and M.Z.S. collected and analysed the behavioural data. Fig. 1A and fig. 1B were drawn by Y.N. Wang. Fig. 1C was drawn by M.Z. Sun. Fig. 1D was drawn by Y.Y. Hou. All authors discussed the results and implications and commented on the manuscript at all stages.

\section{Additional information}

Supplementary information accompanies this paper at http://www.nature.com/ scientificreports

Competing financial interests: The authors declare no competing financial interests. How to cite this article: Wang, Y.-N. et al. Behavioural screening of zebrafish using neuroactive traditional Chinese medicine prescriptions and biological targets. Sci. Rep. 4 5311; DOI:10.1038/srep05311 (2014)

This work is licensed under a Creative Commons Attribution-NonCommercialNoDerivs 4.0 International License. The images or other third party material in this article are included in the article's Creative Commons license, unless indicated otherwise in the credit line; if the material is not included under the Creative Commons license, users will need to obtain permission from the license holder in order to reproduce the material. To view a copy of this license, visit http:// creativecommons.org/licenses/by-nc-nd/4.0/ 